\title{
Interactive comment on "Investigation of observational error sources in multi Doppler radar vertical air motion retrievals: Impacts and possible solutions" by Mariko Oue et al.
}

\section{Anonymous Referee \#3}

Received and published: 30 January 2019

\section{Overarching Comments}

This paper describes a rigorous evaluation of the North et al. (2017) 3DVAR multi-Doppler air motion retrieval using a high resolution cloud resolving model and a radar-model simulator and carefully examining selected common sources of uncertainty: characteristics of the scanning strategy, time updates of the scanning strategy, and advection corrections to the data. The novel aspect of the paper is that the experiments can control for many aspects of the sources of uncertainty given the high temporal resolution of the model output which can be subsampled nearly arbitrarily for comparison with the retrievals. The paper makes the case that for 
some characteristics of estimating vertical velocity in triple-Doppler networks, that having a Doppler radar capable of scanning a PPI in less than 2 minutes is desirable, particularly for accurately estimating the characteristics of weak updrafts.

One major comment I have is that while the paper's methodology is thorough and the figures are clear and illustrative, the presentation of the results is done in an illogical fashion and does not allow for an easy read. The text refers to many multi-panel figures in a repeated fashion that lead the reviewer to do a lot of page flipping. I suggest breaking up the Figures 5,6,7, and 8 into different figures that follow the logical flow of Section 3 without having to refer back to the figure subpanels over and over again. Alternatively, discussion of the variables in Figs. 5-8 could be done sequentially by retrieved variable rather than by observing strategy. I believe that the former strategy would be easier for the authors to do.

I believe the paper is acceptable for publication with these minor revisions in mind.

\section{Specific Comments}

1. Should the title be revised to include 3DVAR retievals? The paper does not include other traditional dual-Doppler retrievals such as in CEDRIC (which have different assumptions about integrating the continuity equation than 3DVAR), or more advanced analyses such as in SAMURAI (Bell et al.). Different techniques could fare better or worse than the 3DVAR technique.

2. It should be stated in the methdology that this analysis is based on a single case, and performance assessment could vary for different storm characteristics and propagation speeds. For example, a slow moving tropical squall line in low 
vertical wind shear could present less of a challenge for multi-Doppler retrievals than the highly-sheared May 20 MC3E squall line.

3. P5, L3, and elsewhere: the apostrophe is unnecessary.

4. P5, L25: suggest removing the word 'at'.

Interactive comment

5. P6, L15-16: the CRSIM simulates the phase shift and the differential phase shift, not the phase and specific differential phase, correct?

6. P7, L8: An analysis domain top of $10 \mathrm{~km}$ does not top the storm, correct? This would not allow for correct estimation of the storm-top divergence properly in the interpolated simulated radar data. Does this lead to some of the uncertainty unnecessarily?

7. P8, L20: "not unfolded correctly observed Doppler velocity" needs rewording.

8. P9, L14: "at" should be in.

9. P13, L2: The tilt would depend on the propagation speed and the vertical wind shear, which in some regimes (especially in the tropics) could be smaller than in this case.

10. P13, L11: The smoothness function is a Cressman or Barnes filter? If not, what equation did you use?

11. P14, L1: Suggest inserting "potentially" before "large".

12. P14, L25-26: “...but it tends to have higher uncertainty in the areas around the location of strong convection..." - this seems highly subjective. Can you make a quantitative statement related to this topic?

Printer-friendly version

Discussion paper 
13. P15, L7: Regarding an updraft core defined as being larger than $0.5 \mathrm{~km}^{2}$ : Is this at a single level? This is one single pixel, correct? Since the retrieval has a resolution that is effectively 4-6 times the grid spacing, would one expect it to retrieve such small updraft features? Perhaps it is not surprising then that the retrievals have such issues resolving updrafts (particularly weak ones) that are single pixels in the grid? Perhaps increasing this threshold to have larger-sized updrafts (say larger than 2-3 $\mathrm{km}^{3}$ ) would reveal different uncertainties.

14. P15, L16: Suggest inserting an "A" before "Noticable".

15. P16, L8-10: This sentence is awkward.

16. P20, L12-13: "cloud evolution cannot maintain the instantaneous cloud structures": Can you be more specific what you mean by this?

17. P22, L6: "As previous literature has pointed out..."

18. P22, L8: $4.5 \mathrm{~km}$ is mid-levels, no?

19. P22, L16: Need comma after "i.e."

20. P22, L18: "inferior" should be "lower"?

21. P22, L28: Suggest replacing "hard" with "challenging".

22. P23, L10: Scott G.'s last name is misspelled.

23. P23, L20: "DOW's" should be "Doppler on Wheels mobile radars"

Interactive comment on Atmos. Meas. Tech. Discuss., doi:10.5194/amt-2018-442, 2018. 